\title{
Investigating the Correlation between Mathematical Pre-Knowledge and Learning Gains in Service Physics
}

\author{
J M Buick \\ Physics and Electronics, University of New England, Armidale, NSW 2351, Australia \\ E-mail: jbuick@une.edu.au
}

\begin{abstract}
An investigation was undertaken into the relationship between the initial mathematics knowledge of students and their success in a service physics course aimed at science students who require an understanding of basic physics concepts but who are not studying for a physics major. An appropriate method for performing this investigation was developed, implemented and analysed. Results from a small, self-selected sample indicated a correlation between the initial mathematical knowledge of students and learning gains obtained during the course.
\end{abstract}

PACS numbers: 01.40.Fk

Submitted to: Eur. J. Phys.

\section{Introduction}

There is a clear link between physics and mathematics and it is essential that a physics student has a suitable mathematical background. This is an issue for physics students but is generally not considered to be so significant for service physics students since the level of mathematics they require is less. A link between mathematical ability and achievement in physics has recently been studied. Meltzer [1] identified a correlation between mathematical ability at the start of a first-year physics course and the normalised learning gains of the students. It was also found that the normalised learning gains in physics were independent of the initial physics knowledge of the students. The investigation was aimed at the conceptual learning gains of the students, not their ability to handle the mathematics in the course. That is, small normalised learning gain could be associated with students who failed to grasp the concepts rather than student who could not cope with the mathematics. The underlying explanation of these results is not clear; however, they indicate that a physics student is better served by a good pre-knowledge of mathematics rather than a good pre-knowledge of physics, certainly in terms of their learning gains which, over a full degree course, will be the important variable. The aim of this work is to investigate to what extent the findings of Meltzer [1] can be applied to service physics courses where very little pre-knowledge is required by a student. If the findings of Meltzer [1] are mirrored in a service physics course, where an understanding of 
the concepts is considered more important than establishing a mathematical theory, it may be necessary to re-think the level of background mathematics provided during the course or required prior to starting.

\subsection{The Service Physics Course}

The service physics course considered here is a first-year university course for science students, typically from biomedical sciences. It is designed for students with little or no background in physics or mathematics. The mathematics required in the course is covered at the start; this consists of re-arranging equations and the concept of a vector. The course is taught using a traditional lecture, tutorial and laboratory structure. The course is assessed through an exam with a $70 \%$ weighting in which approximately half the questions have a numerical requirement; the remainder of the questions involve descriptive answers. The other $30 \%$ of the assessment consists of assignments submitted during the course and laboratory work.

\subsection{Assessing Mathematical Pre-Knowledge}

To investigate the relationship between learning gains and mathematical pre-knowledge it is necessary to gauge mathematical pre-knowledge. A mathematics test at the start of the course was used to measure the student's mathematical pre-knowledge. This gives a direct measurement of the student's mathematical pre-knowledge at that time and is the method applied by Meltzer [1] and others [2, 3, 4].

\subsection{Developing a Mathematical Pre-Knowledge Test}

A mathematics pre-knowledge test (MPT) must be designed such that it gives a true measure of the range of ability in the course. For example, a test consisting of trivial questions that every student can answer will not reveal any information. A well designed test must contain questions covering the range of student abilities. Without the ability to 'test the test', the questions must be selected based on a general idea of the level of the mathematical preknowledge of the students. In order to ensure the validity of the questions, it was decided to select questions used in other investigations $[1,5,6,7]$. These questions were used in preknowledge tests of physics students, so it might be expected that the service physics students would score less well; however it was felt that a full range of abilities would still be covered. It was decided that using well-established question that had been used in other pre-knowledge tests offset the possible disadvantage arising from the fact that some questions may be at too high a level for the average student in the course.

\section{Assessing Learning Gains in Service Physics}

Once the mathematical pre-knowledge had been measured, it is necessary to measure the learning gains in the service physics course. Following Meltzer [1] this was achieved by a 
series of questions on the topics covered in the course. The questions were answered by the students prior to the course (at the same time as the MPT) and again after the material had been covered. Unlike the MPT test, no questions were available in the literature at a suitable level for the material covered in the course. McDermott and Redish [8] identified a number of research works dealing with students undertaking courses in fluid mechanics (the course topic), however, none deal with the level and topics of this service physics course. It was therefore necessary to build a set of questions which covered the correct material at a level suitable for measuring learning gain. The learning gain can be defined as the difference between a student's results in the service physics post test (SPoT) and the service physics pre test (SPrT), where the questions used in the two tests are the same.

\subsection{Normalised Learning Gains}

The learning gain defined above, gives a measure of how much students have learnt in the course. It is not, however, a good unit of measurement for the success of a student since it discriminates against students who had a good pre-knowledge and benefits students with a poor pre-knowledge. To overcome this Meltzer [1] considered the normalised learning gain, $g$. This is defined as $g=(\mathrm{SPoT} \%-\mathrm{SPrT} \%) /(100-\mathrm{SPrT} \%)$, where SPoT\% and SPrT\% are the percentage scores in the post and pre service physics tests respectively. That is, the increase in the number of questions answered correctly normalised by the maximum possible increase.

\section{Administering the Tests}

The tests were presented in the form of two multiple choice tests: the first consisted of the MPT and the SPrT while the second was the SPoT, consisting of the same questions as the SPrT. Each question paper included an identification number which the students were asked to mark on their answer sheet. Further they were asked to keep a copy of the identification number with their notes so that they could place it on the post test answer sheet. This enabled the learning gain to be calculated while keeping the system anonymous. The students were in the first or second year of a non-physics degree and fell into the 18-22 age group. The class size was 75 students and a self-selected subset of 15 students complete both the pre and post tests. Since the test was anonymous and not compulsory, it was not possible to determine any details about the makeup of this subset.

\section{Analysis of the Test Questions}

It is important that the range of difficulty in the tests span the ability of the students. The same questions were being used for the SPrT and SPoT, so these questions must cover both ranges. This is important here since the SPT questions have not been used previously and the MPT questions were originally designed for physics students rather than service physics students. This was investigated using Rasch Modelling. 
Table 1. Illustration of the Rasch model for 4 students and 6 multiple choice questions. The answers to the questions are represented by a ' 1 ' for correct and a ' 0 ' for wrong.

\begin{tabular}{lccccccc}
\hline & \multicolumn{4}{c}{ Question } & & \multirow{2}{*}{ Total } \\
& 1 & 2 & 3 & 4 & 5 & 6 & \\
\hline Student 1 & 1 & 1 & 1 & 0 & 1 & 0 & 4 \\
Student 2 & 1 & 1 & 1 & 0 & 1 & 0 & 4 \\
Student 3 & 1 & 1 & 0 & 0 & 1 & 0 & 3 \\
Student 4 & 1 & 1 & 0 & 0 & 1 & 0 & 3 \\
\hline Answered correctly, $N$ & 4 & 4 & 2 & 0 & 4 & 0 & \\
\hline
\end{tabular}

\subsection{Rasch Modelling}

Here we analyse the questions, after the tests have been completed, to investigate to what extend the original aims were achieved. This was done using Rasch Modelling [9, 10]. The basic principle is illustrated in table 1 . The first 5 rows represent a spreadsheet recording the marks of 4 students answering 6 multiple choice questions. The total is also given and further statistics might be applied; for example the class average is 3.5 and the standard deviation is 0.5 . The principle behind Rasch modelling is to apply the same approach to analyse the questions to see if their difficulty matches the ability of the students. In the bottom row of table 1, the number of times each question has been answered correctly, $N$, is displayed. We see that each student answered questions 1, 2 and 5 correctly. This suggests that they are too easy, relative to the ability of the students, and have no effect in separating the students. In the same way questions 4 and 6 were always answered incorrectly and are too difficult, relative to the students' ability. Thus only question 3 is suitable for separating the students' ability. That is, if only question 3 had been asked the results would be the same (in terms of rank order). In Rasch Modelling the students' responses are used to determine the difficulty, $\delta_{i}$, of each question and the ability, $\beta_{n}$, of each student. Knowing $\beta_{n}$ and $\delta_{i}$ for each question and student does not determine a result table (such as table 1) since a student with a high (low) ability will occasionally answer an easy (hard) question incorrectly (correctly). Thus a probabilistic approach is employed in Rasch analysis where the probability of Student $\mathrm{Sa}$ answering question $\mathrm{Qb}$ correctly is

$$
\frac{e^{\beta} \mathrm{Sa}^{-\delta} \mathrm{Qb}}{1+e^{\beta} \mathrm{Sa}^{-\delta} \mathrm{Qb}} \text {. }
$$

This is the basis of Rasch Modelling. Here it was performed using Ministep [11]. The outcomes for the tests are presented in figure 1 and will be discussed in sections 4.2 and 4.3; here only the interpretation of the output will be considered. The horizontal line separates the students (represented by the numbers in diamonds below the line) from the questions (represented by numbers in squares above the line). Also shown is a Gaussian curve. This is included to indicate the positions of the mean and standard deviation calculated in the analysis. It is not intended to infer that the data has a Gaussian form. The units on the horizontal axis $(-2,-1, \ldots)$ are in logits. This is a scale that can be applied equally to both sets (students 


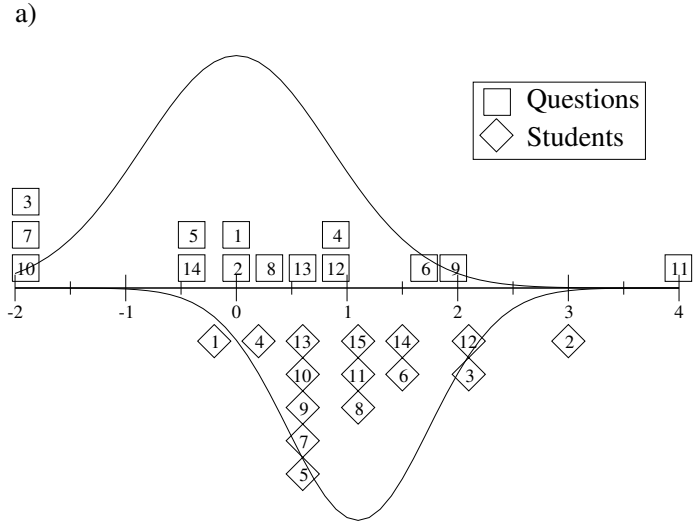

b)

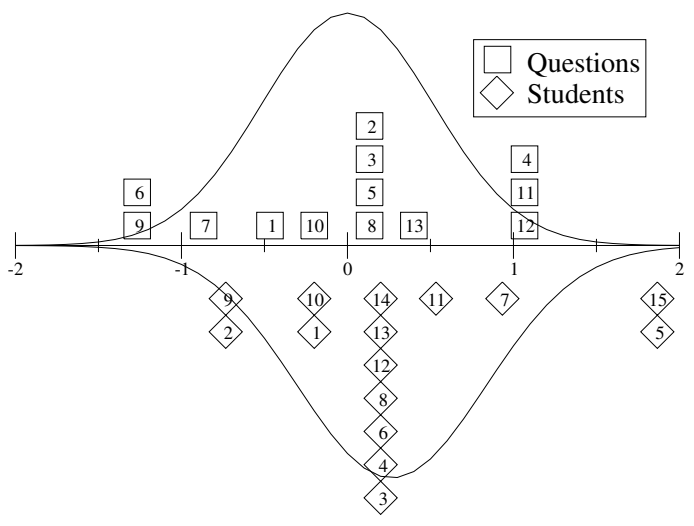

c)

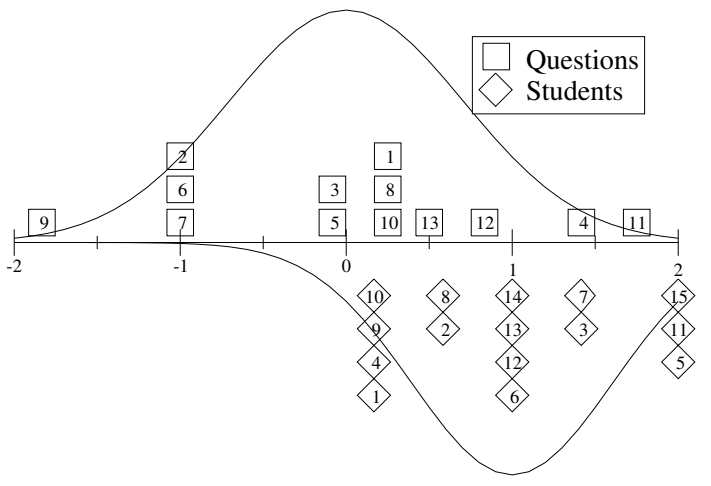

Figure 1. Rasch analysis of a) MPT, b) SPrT and c) SPoT.

and questions) and is linear. An increasing value for the students represents increased ability while for the questions increased difficulty. The logits, or $\log$ odds are the difference between student ability and question difficulty $\left(\beta_{\mathrm{Sa}}-\delta_{\mathrm{Qb}}\right.$ in (1)). The zero point in the logit scale corresponds to the mean of question difficulty. Whenever a student, Sa, and a question, Qb, line up; student Sa had a 50\% chance of getting question $\mathrm{Qb}$ (or a question with the same level of difficulty) correct. This student will have a less than $50 \%$ chance of getting the right answer for a question to the right of $\mathrm{Qb}$ in the figure, the probability of a correct answer decreasing with the increasing position of the question (difficulty). Similarly Sa will have a greater than $50 \%$ chance of answering a question to the left of $\mathrm{Qb}$ in the figure. A student who has a 50\% chance of answering a question of mean difficulty correctly, therefore, has a logit value of 0 .

\subsection{Rasch Modelling of the MPT}

Rasch analysis of the MPT is shown in figure 1a. It is evident that there is a mismatch between the difficulty of the questions and the students' ability. The zero corresponds to a question of mean difficulty (Q1 and Q2), however the mean position for the students is greater than one. In other words, the questions were too easy for the students, or the students' ability was too 
great for the questions. This is slightly surprising since an initial assumption was that the students had trouble with aspects of mathematics. It may be that students who felt they could answer most questions submitted their answers, while those who found it difficult were less inclined to finish or hand in. This must be considered when interpreting the final results. Despite this discrepancy in matching the difficulty of the questions to the student's ability, the questions span the range of the student ability with Q11 difficult enough to cause problems for the most able students and Q5 and Q14 easy enough that the least able students have a reasonable probability of answering them correctly. Between these questions there is a fairly even spread of questions. Q3, Q7 and Q10 are wasted since they give little or no information which can be used to split the student's ability. Ideally they should be replaced with harder questions if the tests were to be reused. Despite these flaws the Rasch modelling suggests that the questions are generally suitable for the students and can be used to assess their preknowledge in mathematics as required.

\subsection{Rasch Modelling of the SPrT and SPoT}

Rasch analysis was also performed for both the pre and post service physics tests and is shown in figure $1 \mathrm{~b}$ and $\mathrm{c}$ respectively. In figure $1 \mathrm{~b}$ the difficulty of the questions and the students' ability are reasonably well matched. Q4, Q11 and Q12 are bunched at the top of the diagram suggesting they are particularly difficult. In figure1c questions are not so well matched to the student with the mean for the student's ability occurring at the mean plus one standard deviation for the question difficulty. This is to be expected if there is an increase in the student's ability due to taking the course. This is also seen from the six questions, almost half, which fall below the lowest student level. It is also interesting to note that the higher level of student ability has acted to separate Q4, Q11 and Q12. There is also some change in the order of difficulty of the questions. This is possibly due to certain aspects of the course being understood better than others. Thus the Rasch analysis indicates that the difficulty of the questions is reasonably well matched to the students' ability making them suitable for their intended use.

\section{Learning Gains in Service Physics}

The normalised learning gain $g$ is shown in figure 2 as a function of the SPrT results. No positive correlation was found and in fact the best-fit line has a small negative gradient. Figure 3 shows $g$ plotted against the MPT results. For comparison between figure 3 and the results of Meltzer [1] we note that the correlation coefficient quoted by Meltzer [1] is $r=+0.46$ and the best-fit straight line (in the units used here) is $g=0.00568 M+0.228$. Comparing the results here with the results of Meltzer[1] indicates a number of similarities. Neither set of results shows a correlation between normalised gain and pre-knowledge of the subject. The results in figure 3 indicate a positive correlation with $r=+0.51$, similar to the value found by Meltzer [1]. The value of the gradient for the best-fit straight line is also similar although it is difficult to determine the significance of this given the large difference in the constant term in both 


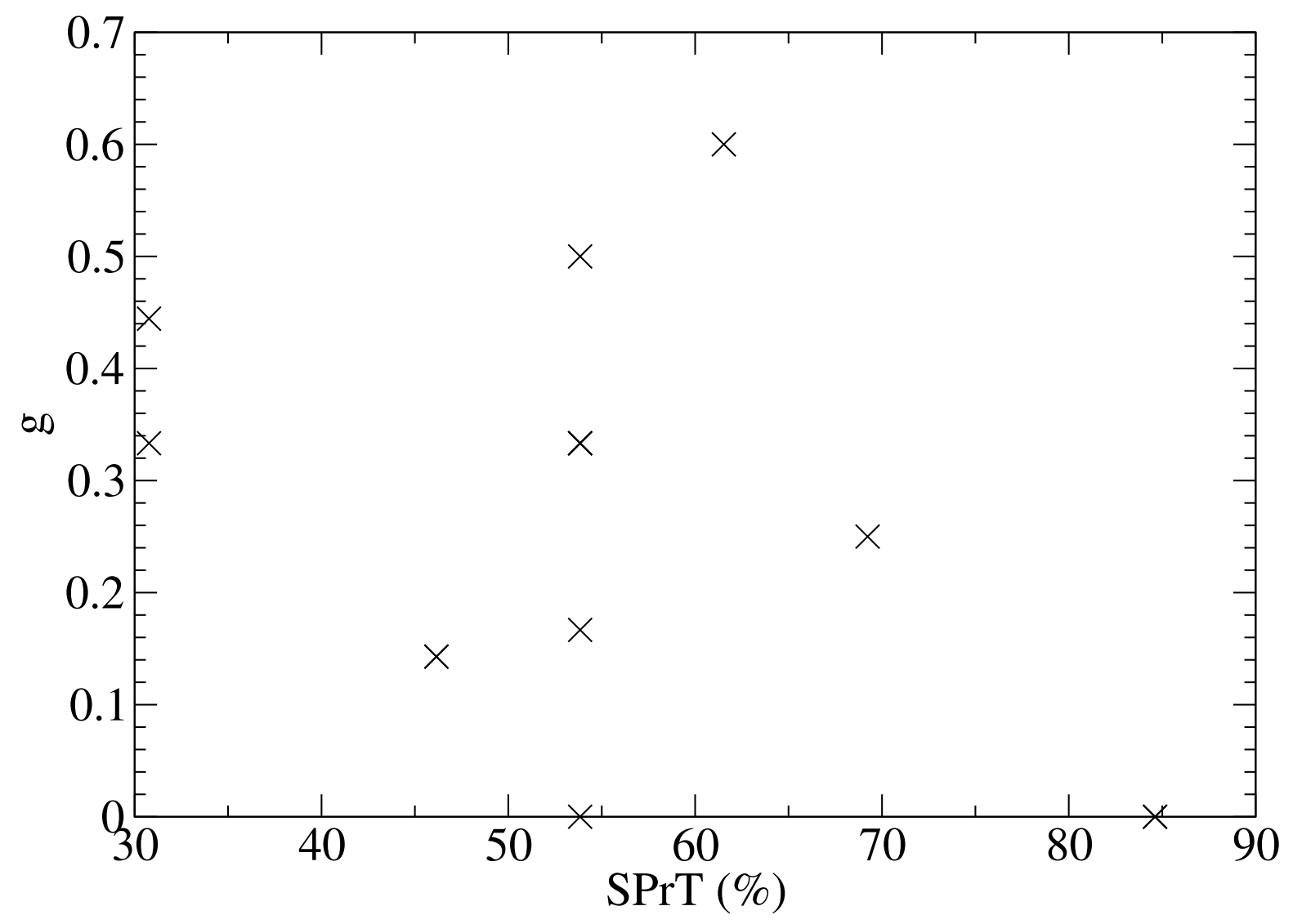

Figure 2. The normalised learning gain and the SPrT results.

equations. Given the relatively small number of responses to both tests in the present research (15), it is not possible to give too much weight to the findings. Despite the small number of participants, the results in figure 3 do indicate a positive correlation between the normalised learning gains of service physics students and their pre-knowledge of mathematics, similar to the results for physics students reported by Meltzer[1].

It is also important to consider the subset of students who are represented in this study. This subset contained only $20 \%$ of the class and was self-selected. Due to the anonymous nature of the study it was not possible to determine if the subset is representative of the whole class, or the reasons why students chose not to participate. It is possible that the students who had difficulty with the questions would be less likely to completed and submit the tests. There is no direct evidence for this; however, the relatively high marks obtained in the MPT by students with a limited mathematical background are consistent with this hypothesis. Since only a small proportion of the class participated in the study it is clear that the self-selection mechanism will introduce a bias to the results and any conclusion drawn from this study may not apply to the whole class. Without knowledge of which students completed the tests it is only possible to speculate as to the make of this group. Thus the conclusions of this study 


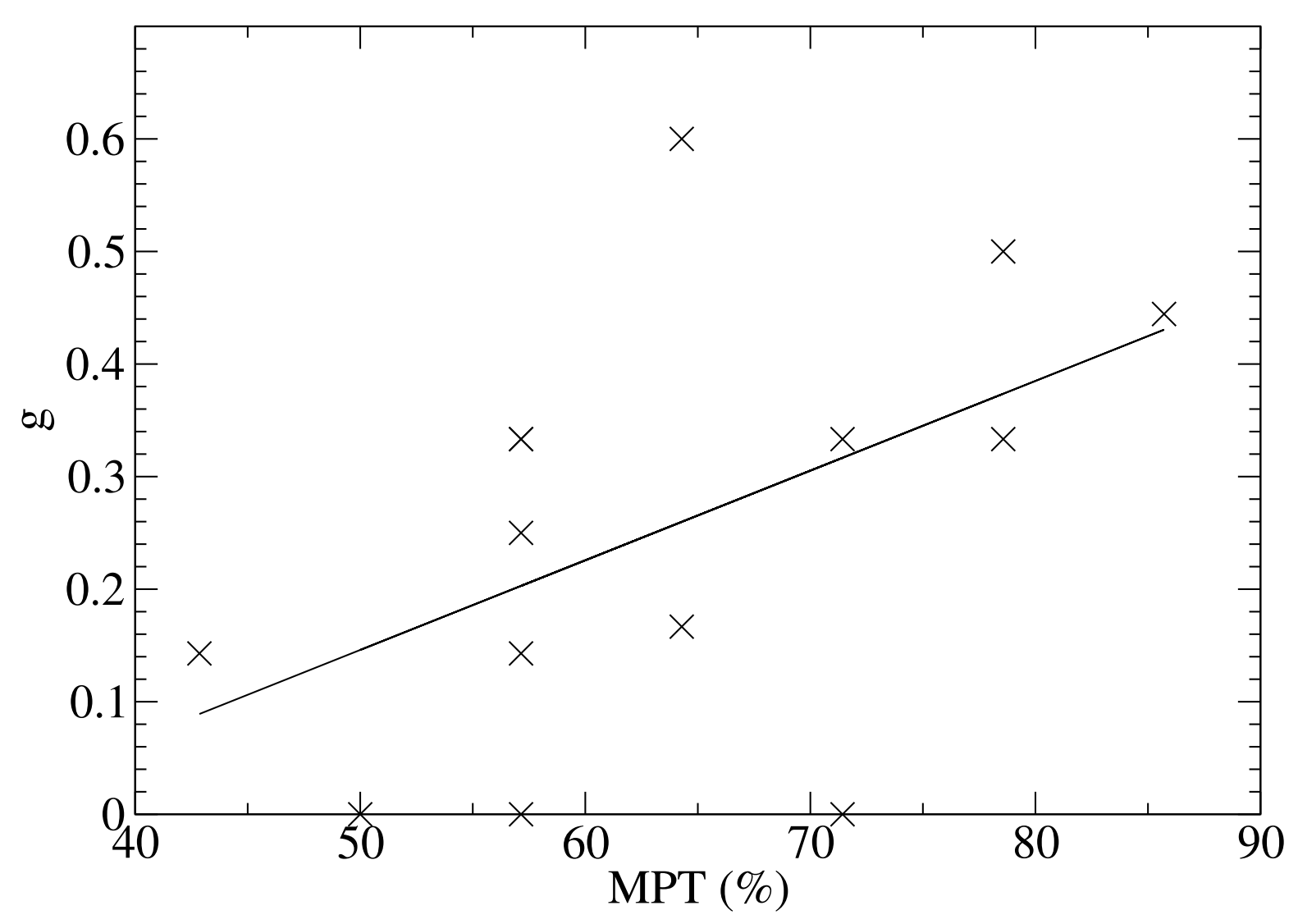

Figure 3. The normalised learning gain and the MPT results. The best-fit line is $g=0.0080 M$ -0.25 , where $M$ is the percentage MPT mark. The correlation coefficient is $r=+0.51$.

may not be applicable to all students in service physics courses; however, even if it applies to only $20 \%$ of such students, consideration of the results by those involved in teaching service physics could lead to improved learning gains for a significant number of students.

\section{Discussion and Conclusions}

The results in figure 3 indicate a correlation between learning gains in service physics and preknowledge of mathematics. This conclusion is drawn from a limited number of data points (15). The results suggest a positive correlation, however, the small number of responses indicates a level of doubt in the certainty of this conclusion. It is also evident that the results only represent the subset of the class who self-selected to undertake the survey and may not be representative of the whole class. At the very least the results indicate that there may be a correlation and that it would be advantageous to perform a further, larger study. In such a study it would be essential that students with a lower level of mathematical ability were included. The results of the Rasch analysis presented in figure 1 could be used to ensure that 
suitable questions were asked. For example questions Q3, Q7 and Q10 could be replaced with more difficult questions.

It is also interesting to compare figures. $1 \mathrm{~b}$ and $\mathrm{c}$. By considering the level of difficulty of the questions it is possible to identify in which topic areas the students understanding has improved. We note that the numerical logit scales vary between the two figures due to the learning gains experienced by the students. Thus we can not compare values between the two figures. We can, however, place the questions in rank order of difficulty and look for questions which have moved out of place. In this study Q1 and Q10 have both increased in relative difficulty. This suggests that students are having difficulty with the concepts in these questions. Thus the Rasch modelling has also enabled two topics with a low learning gain to be identified. This is probably due to local aspects of the teaching and not a global issue. It does, however, highlight how such topics can be identified in the Rasch analysis so that the manner in which they are covered can be modified.

In conclusion, a template for comparing the learning gains of non-physics science students taking a service physics course with the student's pre-knowledge of mathematics has been developed and analysed. Further, results obtained using the template suggest that there is a correlation between learning gain and mathematical pre-knowledge. This mirrors the results of Meltzer [1] for students studying toward a physics major. A larger survey is required to provide a greater level of statistical certainty.

\section{Acknowledgments}

The author would like to thank the survey participants; and also Robyn Smyth, Sarah Stein, and the members of PDAL496, 2004 for useful comments and discussions.

\section{References}

[1] D.E. Meltzer. The relationship between mathematics preparation and conceptual learning gains in physics: A possible "hidden variable" diagnostic pretest scores. Am. J. Phys., 70:1259, 2002.

[2] I. Johnston and R. Millar. Is there a right way to teach physics? CAL-laborate, 5, 2000.

[3] I.A. Halloun and D. Hestenes. The initial knowledge state of college physics students. Am. J. Phys., $53: 1043,1985$.

[4] N. Emarat and I. Johnston. The effectiveness of the Thai traditional teaching in the introductory physics course: A comparison with the US and Australian approaches. CAL-laborate, 9, 2002.

[5] H.T. Hudson and R.M. Rottmann. Correlation between performance in physics and prior mathematics knowledge. J. Res. Sci. Teach., 18:291, 1981.

[6] H.T. Hudson. A comparison of mathematics backgrounds between american and south african physics students. Sci. Educ., 73:459, 1989.

[7] W. Wollman and F. Lawrenz. Identifying potential "dropouts" from college physics classes. J. Res. Sci. Teach., 21:385, 1984.

[8] L.C. McDermott and E.F. Redish. Resource letter: Per-1: Physics education research. Am. J. Phys., 67:755, 1999.

[9] T.G. Bond and C. M. Fox. Applying The Rasch Model: Fundamental Measurements in the Human Sciences. Lawrence Erlbaum Associated, London, 2001.

[10] I.W. Fischer, G.H.and Molenaar. Rasch Models: Foundations, Recent Developments, and Applications. Springer-Verlag, New York, 1995. 
[11] http://www.winsteps.com/ministep.htm. 